\title{
CHEBYSHEV TYPE INEQUALITIES FOR CONFORMABLE FRACTIONAL INTEGRALS
}

\author{
ERHAN SET, AHMET OCAK AKDEMIR, AND İLKER MUMCU
}

Received 29 November, 2018

\begin{abstract}
In this article, firstly some necessary definitions and results involving fractional integrals are given. Secondly, a new identity involving conformable fractional integrals is given. Then, by using this identity, we establish new Chebyshev inequalities for the Chebyshev functional via conformable fractional integral.
\end{abstract}

2010 Mathematics Subject Classification: 26A33; 26D10; 26D15; 33B15.

Keywords: Chebyshev inequality, Riemann-Liouville fractional integral, conformable fractional integrals

\section{INTRODUCTION}

There are many different type integral inequalities in the literature such as classical and analytic inequalities. We will start with the most prominent one of classical inequalities that is called Chebyshev inequality.

This inequality was established by Chebyshev in [3] as following;

$$
|T(f, g)| \leq \frac{1}{12}(b-a)^{2}\left\|f^{\prime}\right\|_{\infty}\left\|g^{\prime}\right\|_{\infty},
$$

where $f, g:[a, b] \rightarrow \mathbb{R}$ are absolutely continuous functions whose derivatives $f^{\prime}, g^{\prime} \in$ $L_{\infty}[a, b]$ and

$$
T(f, g)=\frac{1}{b-a} \int_{a}^{b} f(x) g(x) d x-\left(\frac{1}{b-a} \int_{a}^{b} f(x) d x\right)\left(\frac{1}{b-a} \int_{a}^{b} g(x) d x\right),
$$

which is called the Čebyšev functional, provided the integrals in (1.2) exist.

We will remind some well-known concepts as followings:

The beta function defined as follows [5, p18]:

$$
B(a, b)=\frac{\Gamma(a) \Gamma(b)}{\Gamma(a+b)}=\int_{0}^{1} t^{a-1}(1-t)^{b-1} d t, \quad a, b>0,
$$

where $\Gamma(\alpha)=\int_{0}^{\infty} e^{-t} u^{\alpha-1} d u$ is Gamma function. 
Definition 1. Let $f \in L_{1}[a, b]$. The Riemann-Liouville integrals $J_{a+}^{\alpha} f$ and $J_{b-}^{\alpha} f$ of order $\alpha>0$ are defined by

$$
J_{a+}^{\alpha} f(x)=\frac{1}{\Gamma(\alpha)} \int_{a}^{x}(x-t)^{\alpha-1} f(t) d t, \quad x>a
$$

and

$$
J_{b-}^{\alpha} f(x)=\frac{1}{\Gamma(\alpha)} \int_{x}^{b}(t-x)^{\alpha-1} f(t) d t, \quad x<b
$$

respectively. Here $J_{a+}^{0} f(x)=J_{b-}^{0} f(x)=f(x)$.

In [2], Belarbi and Dahmani established following theorems for the Chebyshev inequalities.

Theorem 1. Let $f$ and $g$ be two synchronous functions on $[0, \infty)$. Then for all $t>0, \alpha>0$, we have:

$$
J^{\alpha}(f g) \geq \frac{\Gamma(\alpha+1)}{t^{\alpha}} J^{\alpha} f(t) J^{\alpha} g(t) .
$$

Theorem 2. Let $f$ and $g$ be two synchronous functions on $[0, \infty)$. Then for all $t>0, \alpha>0, \beta>0$, we have:

$$
\frac{t^{\alpha}}{\Gamma(\alpha+1)} J^{\beta}(f g)(t)+\frac{t^{\beta}}{\Gamma(\beta+1)} J^{\alpha}(f g)(t) \geq J^{\alpha} f(t) J^{\beta} g(t)+J^{\beta} f(t) J^{\alpha} g(t)
$$

Theorem 3. Let $\left(f_{i}\right)_{i=1, \ldots, n}$ be $n$ positive inreasing functions on $[0, \infty)$. Then for any $t>0, \alpha>0$, we have

$$
J^{\alpha}\left(\prod_{i=1}^{n} f_{i}\right)(t) \geq\left(J^{\alpha}(1)\right)^{1-n} \prod_{i=1}^{n} J^{\alpha} f_{i}(t .)
$$

Theorem 4. Let $f$ and $g$ be two functions defined on $[0, \infty)$, such that $f$ is increrasing, $g$ is differentiable and there exist a real number $m:=i n f_{t \geq 0} g^{\prime}(t)$. Then the inequality

$$
J^{\alpha}(f g)(t) \geq\left(J^{\alpha}(1)\right)^{-1} J^{\alpha} f(t) J^{\alpha} g(t)-\frac{m t}{\alpha+1} J^{\alpha} f(t)+m J^{\alpha}(t f(t))
$$

is valid for all $t>0, \alpha>0$.

In [4], Khalil et al. define a new well-behaved simple fractional derivative called conformable fractional derivative depending just on the basic limit definition of the derivative. They also defined the fractional integral of order $0<\alpha \leq 1$ only.

In [1], Abdeljawad gave the definition of left and right conformable fractional integrals of any order $\alpha>0$. 
Definition 2. Let $\alpha \in(n, n+1]$ and set $\beta=\alpha-n$ then the left conformable fractional integral starting at $a$ if order $\alpha$ is defined by

$$
\left(I_{\alpha}^{a} f\right)(t)=\frac{1}{n !} \int_{a}^{t}(t-x)^{n}(x-a)^{\beta-1} f(x) d x
$$

Analogously, the right conformable fractional integral is defined by

$$
\left({ }^{b} I_{\alpha} f\right)(t)=\frac{1}{n !} \int_{t}^{b}(x-t)^{n}(b-x)^{\beta-1} f(x) d x .
$$

In [7], Set and Mumcu gave following Lemma

Lemma 1. If $g:[a, b] \rightarrow \mathbb{R}$ is integrable and symmetric to $(a+b) / 2$ with $a<b$, then

$$
I_{\alpha}^{a} g(b)={ }^{b} I_{\alpha} g(a)=\frac{1}{2}\left[{ }^{b} I_{\alpha} g(a)+I_{\alpha}^{a} g(b)\right]
$$

with $\alpha \in(n, n+1]$.

Notice that if $\alpha=n+1$ then $\beta=\alpha-n=n+1-n=1$ and hence $\left(I_{\alpha}^{a} f\right)(t)=$ $\left(J_{n+1}^{a} f\right)(t)$.

For some recent results related to conformable fractional integrals, see, e.g., [6, 7]. The main purpose of this paper is to establish Chebyshev type inequalities for conformable fractional integral.

\section{ChebySheV TYPE INEQUALITIES}

Lemma 2. Let $f:[a, b] \rightarrow \mathbb{R}_{+}$be function, where $0 \leq a<b$. If $f \in L[a, b]$ then we get

$$
\begin{aligned}
& \int_{0}^{1} t^{n}(1-t)^{\alpha-n-1} f(t a+(1-t) b) d t+\int_{0}^{1} t^{n}(1-t)^{\alpha-n-1} f((1-t) a+t b) d t \\
& =\frac{n !}{(b-a)^{\alpha}}\left[{ }^{b} I_{\alpha} f(a)+I_{\alpha}^{a} f(b)\right] .
\end{aligned}
$$

for $\alpha \in(n, n+1], n=0,1,2, \ldots$.

Proof. By integration by parts, we can state

$$
\begin{array}{rl}
\int_{0}^{1} t^{n}(1-t)^{\alpha-n-1} & f(t a+(1-t) b) d t= \\
& =\frac{1}{b-a} \int_{a}^{b}\left(\frac{b-x}{b-a}\right)^{n}\left(\frac{x-a}{b-a}\right)^{\alpha-n-1} f(x) d x \\
& =\frac{1}{(b-a)^{\alpha}} \int_{a}^{b}(b-x)^{n}(x-a)^{\alpha-n-1} f(x) d x \\
& =\frac{n !}{(b-a)^{\alpha}}\left(I_{\alpha}^{a} f\right)(b)
\end{array}
$$


and

$$
\begin{array}{rl}
\int_{0}^{1} t^{n}(1-t)^{\alpha-n-1} & f((1-t) a+t b) d t= \\
& =\frac{1}{b-a} \int_{a}^{b}\left(\frac{x-a}{b-a}\right)^{n}\left(\frac{b-x}{b-a}\right)^{\alpha-n-1} f(x) d x \\
& =\frac{1}{(b-a)^{\alpha}} \int_{a}^{b}(x-a)^{n}(b-x)^{\alpha-n-1} f(x) d x \\
& =\frac{n !}{(b-a)^{\alpha}}\left({ }^{b} I_{\alpha} f\right)(a) .
\end{array}
$$

Adding (2.1) and (2.2), we get desired result.

Theorem 5. Let $f, g:[a, b] \rightarrow \mathbb{R}$ are two monotonic functions of the same monotonicity, $f, g \in L[a, b]$ and $\alpha \in(n, n+1](n=0,1,2, \ldots)$, then the following inequality holds:

$$
\begin{aligned}
& \left.\frac{\Gamma(\alpha+1) \Gamma(\alpha-n)}{2(b-a)^{\alpha}}\right)\left[{ }^{b} I_{\alpha}(f g)(a)+I_{\alpha}^{a}(f g)(b)\right] \\
& \geq \frac{\Gamma(\alpha+1)}{2(b-a)^{\alpha}}\left[{ }^{b} I_{\alpha} f(a)+I_{\alpha}^{a} f(b)\right] \frac{\Gamma(\alpha+1)}{2(b-a)^{\alpha}}\left[{ }^{b} I_{\alpha} g(a)+I_{\alpha}^{a} g(b)\right] .
\end{aligned}
$$

Proof. Our proof is based on the property of positivity of the integral. The basic remark is the inequality

$$
(f(x)-f(y))(g(x)-g(y)) \geq 0
$$

for all $x, y \in[a, b]$. For $x=t a+(1-t) b, t \in[0,1]$ we obtain

$$
(f(t a+(1-t) b)-f(y))(g(t a+(1-t) b)-g(y)) \geq 0 .
$$

Similarly, for $x=(1-t) a+t b, t \in[0,1]$ we have

$$
(f((1-t) a+t b)-f(y))(g((1-t) a+t b)-g(y)) \geq 0 .
$$

Adding the inequalities (2.4) and (2.5), then multiplying both sides with $t^{n}(1-$ $t)^{\alpha-n-1}$ and integrating with respect to $t$ over $[0,1]$ we have:

$$
\begin{aligned}
& \int_{0}^{1} t^{n}(1-t)^{\alpha-n-1}(f g)(t a+(1-t) b) d t \\
& +\int_{0}^{1} t^{n}(1-t)^{\alpha-n-1}(f g)((1-t) a+t b) d t+2 B(n+1, \alpha-n) f(y) g(y) \\
& \geq g(y)\left(\int_{0}^{1} t^{n}(1-t)^{\alpha-n-1} f(t a+(1-t) b) d t\right. \\
& \left.+\int_{0}^{1} t^{n}(1-t)^{\alpha-n-1} f((1-t) a+t b) d t\right)
\end{aligned}
$$




$$
\begin{aligned}
& +f(y)\left(\int_{0}^{1} t^{n}(1-t)^{\alpha-n-1} g(t a+(1-t) b) d t\right. \\
& \left.+\int_{0}^{1} t^{n}(1-t)^{\alpha-n-1} g((1-t) a+t b) d t\right) .
\end{aligned}
$$

We apply Lemma 2 and multiplying both sides by $\frac{1}{n !}$

$$
\begin{aligned}
& \frac{1}{(b-a)^{\alpha}}\left[{ }^{b} I_{\alpha}(f g)(a)+I_{\alpha}^{a}(f g)(b)\right]+\frac{2}{n !} B(n+1, \alpha-n-1) f(y) g(y) \\
& \geq \frac{g(y)}{(b-a)^{\alpha}}\left[{ }^{b} I_{\alpha} f(a)+I_{\alpha}^{a} f(b)\right]+\frac{f(y)}{(b-a)^{\alpha}}\left[{ }^{b} I_{\alpha} g(a)+I_{\alpha}^{a} g(b)\right] .
\end{aligned}
$$

We put $y=t a+(1-t) b, t \in[0,1]$ in $(2.7)$, whence it follows

$$
\begin{aligned}
& \frac{1}{(b-a)^{\alpha}}\left[{ }^{b} I_{\alpha}(f g)(a)+I_{\alpha}^{a}(f g)(b)\right]+\frac{2}{n !} B(n+1, \alpha-n-1)(f g)(t a+(1-t) b) \\
& \geq \frac{g(t a+(1-t) b)}{(b-a)^{\alpha}}\left[{ }^{b} I_{\alpha} f(a)+I_{\alpha}^{a} f(b)\right]+\frac{f(t a+(1-t) b)}{(b-a)^{\alpha}}\left[{ }^{b} I_{\alpha} g(a)+I_{\alpha}^{a} g(b)\right] .
\end{aligned}
$$

On the other hand, for $y=(1-t) a+t b, t \in[0,1]$ in (2.7) we have

$$
\begin{aligned}
& \frac{1}{(b-a)^{\alpha}}\left[{ }^{b} I_{\alpha}(f g)(a)+I_{\alpha}^{a}(f g)(b)\right]+\frac{2}{n !} B(n+1, \alpha-n-1)(f g)((1-t) a+t b) \\
& \geq \frac{g((1-t) a+t b)}{(b-a)^{\alpha}}\left[{ }^{b} I_{\alpha} f(a)+I_{\alpha}^{a} f(b)\right]+\frac{f((1-t) a+t b}{(b-a)^{\alpha}}\left[{ }^{b} I_{\alpha} g(a)+I_{\alpha}^{a} g(b)\right] .
\end{aligned}
$$

Adding the inequalities (2.8) and (2.9), then the multiplying both sides $t^{n}(1-t)^{\alpha-n-1}$ and integrating with respect to $t$ over $[0,1]$, one has

$$
\begin{aligned}
& \frac{2}{(b-a)^{\alpha}} B(n+1, \alpha-n)\left[{ }^{b} I_{\alpha}(f g)(a)+I_{\alpha}^{a}(f g)(b)\right] \\
& +\frac{2}{n !} B(n+1, \alpha-n)\left(\int_{0}^{1} t^{n}(1-t)^{\alpha-n-1}(f g)(t a+(1-t) b)\right. \\
& \left.+\int_{0}^{1} t^{n}(1-t)^{\alpha-n-1}(f g)((1-t) a+t b)\right) \\
& \geq \frac{1}{(b-a)^{\alpha}}\left[{ }^{b} I_{\alpha} f(a)+I_{\alpha}^{a} f(b)\right] \\
& \times\left(\int_{0}^{1} t^{n}(1-t)^{\alpha-n-1} g(t a+(1-t) b) \int_{0}^{1} t^{n}(1-t)^{\alpha-n-1} g((1-t) a+t b)\right) \\
& +\frac{1}{(b-a)^{\alpha}}\left[{ }^{b} I_{\alpha} g(a)+I_{\alpha}^{a} g(b)\right]
\end{aligned}
$$




$$
\times\left(\int_{0}^{1} t^{n}(1-t)^{\alpha-n-1} f(t a+(1-t) b) \int_{0}^{1} t^{n}(1-t)^{\alpha-n-1} f((1-t) a+t b)\right) .
$$

We apply Lemma 2

$$
\begin{aligned}
& \frac{4}{(b-a)^{\alpha}} B(n+1, \alpha-n)\left[{ }^{b} I_{\alpha}(f g)(a)+I_{\alpha}^{a}(f g)(b)\right] \\
& \geq \frac{2 n !}{(b-a)^{2 \alpha}}\left[{ }^{b} I_{\alpha} f(a)+I_{\alpha}^{a} f(b)\right]\left[{ }^{b} I_{\alpha} g(a)+I_{\alpha}^{a} g(b)\right] .
\end{aligned}
$$

Multiplying both sides of the last inequality with $\frac{(\Gamma(\alpha+1))^{2}}{8}$ and using the properties of the beta functions, we obtain desired inequality.

Remark 1. If we take $\alpha=n+1$ in above theorem, then the inequality (2.3) becomes:

$$
\begin{aligned}
& \left.\frac{\Gamma(\alpha+1)}{2(b-a)^{\alpha}}\right)\left[J_{a+}^{\alpha}(f g)(b)+J_{b-}^{\alpha}(f g)(a)\right] \\
\geq & \frac{\Gamma(\alpha+1)}{2(b-a)^{\alpha}}\left[J_{a+}^{\alpha} f(b)+J_{b-}^{\alpha} f(a)\right] \frac{\Gamma(\alpha+1)}{2(b-a)^{\alpha}}\left[J_{a+}^{\alpha} g(b)+J_{b-}^{\alpha} g(a)\right] .
\end{aligned}
$$

Remark 2. In above theorem, if we take $\alpha=n+1, b=t, a=0$ and $f, g$ is symmetric to $(a+b) / 2$, and use Lemma 1 then inequality (2.3) becomes inequality (1.3).

Theorem 6. Let $f, g:[a, b] \rightarrow \mathbb{R}$ are two monotonic functions of the same monotonicity, $f, g \in L[a, b], \alpha \in[n, n+1](n=0,1,2, \ldots)$ and $\beta \in(k, k+1](k=0,1,2, \ldots)$, then

$$
\begin{aligned}
& \frac{2}{(b-a)^{\beta}} B(k+1, \beta-k)\left[{ }^{b} I_{\alpha}(f g)(a)+I_{\alpha}^{a}(f g)(b)\right] \\
& +\frac{2}{(b-a)^{\alpha}} B(n+1, \alpha-n)\left[{ }^{b} I_{\beta}(f g)(a)+I_{\beta}^{a}(f g)(b)\right] \\
& \geq \frac{1}{(b-a)^{\alpha+\beta}}\left[{ }^{b} I_{\alpha} f(a)+I_{\alpha}^{a} f(b)\right]\left[{ }^{b} I_{\beta} g(a)+I_{\beta}^{a} g(b)\right] \\
& +\frac{1}{(b-a)^{\alpha+\beta}}\left[{ }^{b} I_{\alpha} g(a)+I_{\alpha}^{a} g(b)\right]\left[{ }^{b} I_{\beta} f(a)+I_{\beta}^{a} f(b)\right] .
\end{aligned}
$$

Proof. Adding the inequalities (2.8) and (2.9), and then multiplying both sides of obtained inequality with $t^{k}(1-t)^{\beta-k-1}$ and integrating with respect to $t$ over $[0,1]$, we get

$$
\begin{aligned}
& \frac{2}{(b-a)^{\beta}} B(n+1, \beta-n-1)\left[{ }^{b} I_{\alpha}(f g)(a)+I_{\alpha}^{a}(f g)(b)\right] \\
& +\frac{2}{n !} B(n+1, \alpha-n-1)\left(\int_{0}^{1} t^{k}(1-t)^{\beta-k-1}(f g)(t a+(1-t) b)\right.
\end{aligned}
$$




$$
\begin{aligned}
& \left.+\int_{0}^{1} t^{k}(1-t)^{\beta-k-1}(f g)((1-t) a+t b)\right) \\
& \geq \frac{1}{(b-a)^{\alpha}}\left[{ }^{b} I_{\alpha} f(a)+I_{\alpha}^{a} f(b)\right] \\
& \times\left(\int_{0}^{1} t^{k}(1-t)^{\beta-k-1} g(t a+(1-t) b) \int_{0}^{1} t^{k}(1-t)^{\beta-k-1} g((1-t) a+t b)\right) \\
& +\frac{1}{(b-a)^{\alpha}}\left[{ }^{b} I_{\alpha} g(a)+I_{\alpha}^{a} g(b)\right] \\
& \times\left(\int_{0}^{1} t^{k}(1-t)^{\beta-k-1} f(t a+(1-t) b) \int_{0}^{1} t^{k}(1-t)^{\beta-k-1} f((1-t) a+t b)\right) .
\end{aligned}
$$

So, if we use Lemma 2, we have desired result.

Remark 3. Applying Theorem 6 for $\alpha=\beta$, we obtain Theorem 5 .

Remark 4. If we take $\alpha=n+1$ and $\beta=k+1$ in theorem 6, then inequality (2.12) becomes:

$$
\begin{aligned}
& \frac{2}{(b-a)^{k+1}(k+1)}\left[J_{a+}^{\alpha} f g(b)+J_{b-}^{\alpha} f g(a)\right] \\
& +\frac{2}{(b-a)^{n+1}(n+1)}\left[J_{a+}^{\beta} f g(b)+J_{b-}^{\beta} f g(a)\right] \\
& \geq \frac{1}{(b-a)^{n+k+2}}\left[J_{a+}^{\alpha} f(b)+J_{b-}^{\alpha} f(a)\right]\left[J_{a+}^{\beta} g(b)+J_{b-}^{\beta} g(a)\right] \\
& +\frac{1}{(b-a)^{n+k+2}}\left[J_{a+}^{\alpha} g(b)+J_{b-}^{\alpha} g(a)\right]\left[J_{a+}^{\beta} f(b)+J_{b-}^{\beta} f(a)\right] .
\end{aligned}
$$

Remark 5. In above theorem, if we take $\alpha=n+1, b=t, a=0$, and $f, g$ is symmetric to $(a+b) / 2$, and use Lemma 1 , then inequality (2.12) becomes inequality (1.4).

Theorem 7. Let $\left(f_{i}\right)_{i=1, \ldots, n}$ be a positive increasing functions on $[a, b]$. For $\alpha \in$ $(n, n+1] n=0,1,2, \ldots$, we have

$$
\begin{aligned}
& {\left[{ }^{b} I_{\alpha}\left(\prod_{i=1}^{n} f_{i}\right)(a)+I_{\alpha}^{a}\left(\prod_{i=1}^{n} f_{i}\right)(b)\right]} \\
& \geq\left[\frac{\Gamma(\alpha+1)}{2(b-a)^{\alpha} \Gamma(\alpha-n)}\right]^{n-1} \prod_{i=1}^{n}\left[{ }^{b} I_{\alpha}\left(f_{i}\right)(a)+I_{\alpha}^{a}\left(f_{i}\right)(b)\right] .
\end{aligned}
$$

Proof. We prove this theorem by induction. For $n=1$, we have

$$
\left[{ }^{b} I_{\alpha} f_{1}(a)+I_{\alpha}^{a} f_{1}(b)\right] \geq\left[{ }^{b} I_{\alpha} f_{1}(a)+I_{\alpha}^{a} f_{1}(b)\right] \text {. }
$$


For $n=2$, applying Theorem 5, we get

$$
\begin{aligned}
& {\left[{ }^{b} I_{\alpha}\left(f_{1} f_{2}\right)(a)+I_{\alpha}^{a}\left(f_{1} f_{2}\right)(b)\right]} \\
& \left.\geq \frac{\Gamma(\alpha+1)}{2(b-a)^{\alpha} \Gamma(\alpha-n)}\left[{ }^{b} I_{\alpha}\left(f_{1}\right)(a)+I_{\alpha}^{a}\left(f_{1}\right)(b)\right]\left[{ }^{b} I_{\alpha} f_{2}\right)(a)+I_{\alpha}^{a}\left(f_{2}\right)(b)\right] .
\end{aligned}
$$

Now suppose that

$$
\begin{aligned}
& {\left[{ }^{b} I_{\alpha}\left(\prod_{i=1}^{n-1} f_{i}\right)(a)+I_{\alpha}^{a}\left(\prod_{i=1}^{n-1} f_{i}\right)(b)\right]} \\
& \geq\left[\frac{\Gamma(\alpha+1)}{2(b-a)^{\alpha} \Gamma(\alpha-n)}\right]^{n-2} \prod_{i=1}^{n-1}\left[{ }^{b} I_{\alpha}\left(f_{i}\right)(a)+I_{\alpha}^{a}\left(f_{i}\right)(b)\right] .
\end{aligned}
$$

Since $\left(f_{i}\right)_{i=1, \ldots, n}$ are positive increasing functions, then $\left(\prod_{i=1}^{n-1} f_{i}\right)(t)$ is an increasing function. So we can apply Theorem 5 to the functions $\prod_{i=1}^{n-1} f_{i}=g, f_{n}=f$, we obtain

$$
\begin{aligned}
& {\left[{ }^{b} I_{\alpha}\left(\prod_{i=1}^{n} f_{i}\right)(a)+I_{\alpha}^{a}\left(\prod_{i=1}^{n} f_{i}\right)(b)\right]} \\
& \geq\left[\frac{\Gamma(\alpha+1)}{2(b-a)^{\alpha} \Gamma(\alpha-n)}\right] \\
& \times\left[{ }^{b} I_{\alpha}\left(\prod_{i=1}^{n-1} f_{i}\right)(a)+I_{\alpha}^{a}\left(\prod_{i=1}^{n-1} f_{i}\right)(b)\right]\left[{ }^{b} I_{\alpha}\left(f_{n}\right)(a)+I_{\alpha}^{a}\left(f_{n}\right)(b)\right] .
\end{aligned}
$$

Using (2.15), we get desired result.

Remark 6. If we take $\alpha=n+1$ in Theorem 7, then inequality (2.14) becomes:

$$
\begin{aligned}
& {\left[J_{b-}^{\alpha}\left(\prod_{i=1}^{n} f_{i}\right)(a)+J_{a+}^{\alpha}\left(\prod_{i=1}^{n} f_{i}\right)(b)\right]} \\
& \geq\left[\frac{(n+1) !}{2(b-a)^{\alpha}}\right]^{n-1} \prod_{i=1}^{n}\left[J_{a+}^{\alpha} f_{i}(b)+J_{b-}^{\alpha} f_{i}(a)\right] .
\end{aligned}
$$

Remark 7. If we take $\alpha=n+1, b=t, a=0$ and $f_{i} ; i=1, \ldots, n$ is symmetric to $(a+b) / 2$, then, using Lemma 1 , inequality (2.14) becomes inequality (1.5).

Theorem 8. Let $f$ and $g$ be two functions defined on $[0, \infty)$, such that $f$ is increasing, $g$ is differentiable and there exist a real number $m:=i n f_{t \geq 0} g^{\prime}(t)$. Then the inequality

$$
\Gamma(\alpha-n)\left[{ }^{b} I_{\alpha}(f g)(a)+I_{\alpha}^{a}(f g)(b)\right]
$$




$$
\begin{aligned}
& \geq \frac{\Gamma(\alpha+1)}{2(b-a)^{\alpha}}\left[{ }^{b} I_{\alpha} f(a)+I_{\alpha}^{a} f(b)\right]\left[{ }^{b} I_{\alpha} g(a)+I_{\alpha}^{a} g(b)\right] \\
& -\frac{\Gamma(n+2) \Gamma(\beta-n)}{2(b-a)^{\alpha}(\alpha+1)}\left[{ }^{b} I_{\alpha} f(a)+I_{\alpha}^{a} f(b)\right]+m\left[{ }^{b} I_{\alpha}(t f)(a)+I_{\alpha}^{a}(t f)(b)\right] .
\end{aligned}
$$

is valid for all $t>0, \alpha \in(n, n+1], n=0,1,2, \ldots$.

Proof. We consider the function $h(t):=g(t)-m t$. It is clear that $\mathrm{h}$ is differentiable and it is increasing on $[0, \infty)$. Then by using Theorem 5 , we get

$$
\begin{aligned}
& \Gamma(\alpha-n)\left[{ }^{b} I_{\alpha}((g-m t) f)(a)+I_{\alpha}^{a}((g-m t) f)(b)\right] \\
& \geq \frac{\Gamma(\alpha+1)}{2(b-a)^{\alpha}}\left[{ }^{b} I_{\alpha} f(a)+I_{\alpha}^{a} f(b)\right]\left[{ }^{b} I_{\alpha} g(a)+I_{\alpha}^{a} g(b)-m^{b} I_{\alpha} t(a)-m I_{\alpha}^{a} t(b)\right] \\
& \geq \frac{\Gamma(\alpha+1)}{2(b-a)^{\alpha}}\left[{ }^{b} I_{\alpha} f(a)+I_{\alpha}^{a} f(b)\right]\left[{ }^{b} I_{\alpha} g(a)+I_{\alpha}^{a} g(b)\right] \\
& -m B(n+2, \alpha-n) \frac{\Gamma(\alpha+1)}{2(b-a)^{\alpha}}\left[{ }^{b} I_{\alpha} f(a)+I_{\alpha}^{a} f(b)\right] \\
& \geq \frac{\Gamma(\alpha+1)}{2(b-a)^{\alpha}}\left[{ }^{b} I_{\alpha} f(a)+I_{\alpha}^{a} f(b)\right]\left[{ }^{b} I_{\alpha} g(a)+I_{\alpha}^{a} g(b)\right] \\
& -\frac{\Gamma(n+2) \Gamma(\beta-n)}{2(b-a)^{\alpha}(\alpha+1)}\left[{ }^{b} I_{\alpha} f(a)+I_{\alpha}^{a} f(b)\right] .
\end{aligned}
$$

So

$$
\begin{aligned}
& \Gamma(\alpha-n)\left[{ }^{b} I_{\alpha}(f g)(a)+I_{\alpha}^{a}(f g)(b)\right] \\
& \geq \frac{\Gamma(\alpha+1)}{2(b-a)^{\alpha}}\left[{ }^{b} I_{\alpha} f(a)+I_{\alpha}^{a} f(b)\right]\left[{ }^{b} I_{\alpha} g(a)+I_{\alpha}^{a} g(b)\right] \\
& -\frac{\Gamma(n+2) \Gamma(\beta-n)}{2(b-a)^{\alpha}(\alpha+1)}\left[{ }^{b} I_{\alpha} f(a)+I_{\alpha}^{a} f(b)\right]+m\left[{ }^{b} I_{\alpha}(t f)(a)+I_{\alpha}^{a}(t f)(b)\right] .
\end{aligned}
$$

which the proof is completed.

Remark 8. In Theorem 8, if we take $\alpha=n+1$ and $\beta=k+1$, inequality (2.17) becomes:

$$
\begin{aligned}
& \left.\left[J_{a+}^{\beta}(f g)(b)+J_{b-}^{\beta} f g\right)(a)\right] \\
& \geq \frac{(n+2) !}{2(b-a)^{\alpha}}\left[J_{a+}^{\beta} f(b)+J_{b-}^{\beta} f(a)\right]\left[J_{a+}^{\beta} g(b)+J_{b-}^{\beta} g(a)\right] \\
& -\frac{\Gamma(n+2) \Gamma(\beta-n)}{2(b-a)^{\alpha}(\alpha+1)}\left[J_{a+}^{\beta} f(b)+J_{b-}^{\beta} f(a)\right]+m\left[J_{a+}^{\beta} f(b)+J_{b-}^{\beta} g(a)\right] .
\end{aligned}
$$

Remark 9. In Theorem 8, if we take $\alpha=n+1$ and $f, g$ is symmetric to $(a+b) / 2$, then, using Lemma 1 inequality (2.18) becomes inequality (1.6). 


\section{REFERENCES}

[1] T. Abdeljawad, "On conformable fractional calculus," Journal of Computational and Applied Mathematics, vol. 279, pp. 57-66, 2015, doi: 10.1016/j.cam.2014.10.016.

[2] S. Belarbi and Z. Dahmani, "On some new fractional integral inequalities," J. Ineq. Pure Appl. Math., vol. 10, no. 3, pp. Article 86, 5 pp., 2009.

[3] P. Chebyshev, "Sur les expressions approximatives des integrales definies par les autres prises entre les memes limites," Proc. Math. Soc. Charkov, vol. 2, pp. 93-98, 1882.

[4] R. Khalil, M. A. Horani, A. Yousef, and M. Sababheh, "A new definition of fractional derivative," Journal of Computational and Applied Mathematics, vol. 264, pp. 65-70., 2014, doi: 10.1016/j.cam.2014.01.002.

[5] E. Rainville, Special Functions. The Mcmillan Company, New York, 1960.

[6] E. Set, A. Akdemir, and I. Mumcu, "Hadamard's inequality and its extensions for conformable fractional integrals of any order $\alpha>0$," Creat. Math. Inform., vol. 27, no. 2, pp. 197-206, 2018.

[7] E. Set and İ.Mumcu, "Hermite-hadamard-fejer type inequalities for conformable fractional integrals," Miskolc Math. Notes, vol. 20, no. 1, pp. 475-488, 2019, doi: 10.18514/MMN.2019.2421.

\section{Authors' addresses}

\section{Erhan Set}

Ordu University, Department of Mathematics, Faculty of Science and Arts, Ordu, Turkey

E-mail address: erhanset@yahoo.com

\section{Ahmet Ocak Akdemir}

Ağrı İbrahim Çeçen University, Department of Mathematics, Faculty of Science and Arts, Ağrı, Turkey

E-mail address: ahmetakdemireagri.edu.tr

\section{İlker Mumcu}

Ordu University, Department of Mathematics, Faculty of Science and Arts, Ordu, Turkey

E-mail address: mumcuilker@msn.com 